\title{
Raumordnung Schweiz - ein Land, wie wir es wünschen
}

\section{Einleitung}

Vor mehr als vierzig Jahren wurde das Buch «Städte - wie wir sie wünschen» (CAROL und WERNER 1949) veröffentlicht. Dieser Klassiker der schweizerischen Raumplanung soll zum Anlaß genommen werden, zu skizzieren, wie es 1991 um Vorstellungen bezüglich der künftigen räumlichen Ordnung und Organisation unseres Landes steht.

\section{Ausgangslage}

Die schweizerische Raumordnungspolitik ist Ende der achtziger und zu Beginn der neunziger Jahre in Bewegung geraten:

Im Bericht über den Stand und die Entwicklung der Bodennutzung und Besiedlung in der Schweiz vom 14. Dezember 1987 (Raumplanungsbericht 1987) werden die künftigen Aufgaben für die Raumplanung in den nachstehenden fünf Schwerpunkten zusammengefaßt:

1. «Raumplanung muß ernst machen mit der haushälterischen Bodennutzung und eine Trendwende im Bodenverbrauch herbeiführen.

2. Raumplanung muß verstärkt ihre Mittel zur Erhaltung unserer natürlichen Umwelt einsetzen.

3. Raumplanung muß die innere Erneuerung und Ausgestaltung von Siedlungen fördern.

4. Raumplanung muß helfen, unter Einbezug des öffentlichen Verkehrs die Städte als Grundmuster für die dezentralisierte Konzentration funktionsfähig zu erhalten oder wieder funktionsfähig zu machen.

5. Raumplanung muß die Aufgaben der unterschiedlichen Sachbereiche besser aufeinander und auf gemeinsame, von der Bevölkerung getragene räumliche Entwicklungsvorstellungen abstimmen» (Raumplanungsbericht 1987, S. 125).

Im Bericht über die Legislaturplanung 1987-1991 vom 18. Januar 1988 werden folgende vier Stoßrichtungen bezüglich der Raumplanung festgehalten:

1. Erarbeitung eines Realisierungsprogrammes.

2. Anpassung, Revision des Raumplanungsgesetzes und

3. Erarbeitung von Grundzügen der erwünschten räumlichen Entwicklung sowie
4. «Raumplanung und Regionalpolitik besser aufeinander abstimmen und dabei die Anliegen des Umweltschutzes miteinbeziehen» (Legislaturplanung, S. 76).

1989 wurde der im Raumplanungsbericht 1987 und in der Legislaturplanung 1987-1991 angekündigte Bericht über Maßnahmen zur Raumordnungspolitik: Realisierungsprogramm veröffentlicht. «Mit dem Realisierungsprogramm beabsichtigt der Bundesrat: den Vollzug des Raumplanungsgesetzes durch Massnahmen zu stärken, welche

- eine bessere Planung und Koordination der raumwirksamen Tätigkeiten des Bundes herbeiführen,

- die Raumplanung der Kantone fördern und koordinieren helfen und

- die Informationstätigkeit über die Raumplanung und die räumliche Entwicklung verbessern» (Realisierungsprogramm, S. 2).

In diesem Realisierungsprogramm findet sich eine Zusammenstellung der Grundlagen und Planungen des Bundes zur Erfüllung raumwirksamer Aufgaben. Von besonderem Interesse sind die neu zu erarbeitenden Grundlagen und Maßnahmen. Aus Sicht der Geographie dürfte dabei vor allem «Grundzüge der Raumentwicklung» interessieren. In seinem Referat anläßlich der Jubiläumstagung der Schweizerischen Stiftung für Landschaftschutz und Landschaftspflege vom 2. November 1990 in St. Moritz führte der Vorsteher des Eidgenössischen Justizund Polizeidepartementes (EJPD), Bundesrat Arnold Koller, dazu folgendes aus: «. . . mit dem Bericht „Grundzüge der Raumordnung Schweiz” (soll) eine Grundlage für die raumordnungspolitische Koordination der Planungen des Bundes und der Kantone geschaffen werden. Gleichzeitig ist vor dem Hintergrund der europaweiten politischen, gesellschaftlichen und wirtschaftlichen Umwälzungen mit noch unabsehbaren räumlichen Konsequenzen die Frage nach der Zukunft und der Aufgabe des Lebensraumes Schweiz intensiv zu diskutieren» (KOLLER 1990, 10). Fehlt eine solche raumordnungspolitische Gesamtschau, besteht die Gefahr, daß man

Hans Elsasser, Prof. Dr., Geographisches Institut der Universität Zürich-Irchel, Winterthurerstrasse 190, 8057 Zürich. 
sich in der Raumplanung zu stark auf die Lösung von Konflikten beschränkt, die mangels Voraussicht entstanden sind und immer noch entstehen. Die Erarbeitung und breite Diskussion von Grundzügen der räumlichen Entwicklung in der Schweiz könnte ganz wesentlich dazu beitragen, daß der Raumplanung neue Impulse verliehen werden. Am 20. Oktober 1989 ist ferner die revidierte Verordnung über die Raumplanung (RPV) in Kraft getreten. Im Rahmen dieser Revision der Raumplanungsverordnung wurden verschiedene Vorschläge verwirklicht, die von der Expertenkommission zur Revision des Raumplanungsgesetzes erarbeitet wurden. Die Revisionsarbeiten am Bundesgesetz über die Raumplanung (RPG) wurden 1990 eingestellt. Die Revision wurde vor allem von den Kantonen abgelehnt und war in verschiedenen Teilen nicht konsensfähig. Der Bundesrat verzichtet deshalb auf eine Revision des RPG. «Verzicht bedeutet aber keineswegs Nichts-Tun. Ebensowenig gibt ein Verzicht Anlaß zu Besorgnis... So können zur Schließung von Vollzugslücken und zur Überwindung von Vollzugsschwierigkeiten sowie ganz allgemein zur Vereinfachung des Vollzugs umgehend gezielte Anstrengungen im Kompetenzbereich des Bundes unternommen werden. $\mathrm{Zu}$ diesem Zweck ließe sich ein Programm zur Förderung des Vollzugs der Raumplanung („Vollzugsförderungsprogramm RPG”) aufstellen» (KOLLER 1990, 7-8). Dieses Vollzugsförderungsprogramm soll das bereits beschlossene Realisierungsprogramm ergänzen.

Zusammenfassend ist festzuhalten, daß sich die schweizerische Raumordnungspolitik, wie viele andere Politikbereiche auch, aufgrund veränderter Rahmenbedingungen auf nationaler und internationaler Ebene vor einer Neuorientierung steht. «Es geht dabei auch darum, die Begeisterungsfähigkeit für zentrale Fragen der Raumplanung, wie Sicherung und Organisation des Lebensraumes für die Zukunft, die Verbesserung der Lebensqualität, die Verantwortung für künftige Generationen, die gerechte Verteilung von Chancen und Opfern, wieder zu wecken» (RPK 1990, S. 36).

\section{Blick in die Zukunft}

Die heutigen Probleme der räumlichen Ordnung und Organisation der Schweiz sind zum überwiegenden Teil Probleme der Enge. Der begrenzten Bodenfläche, dem knappen Raum stehen stetig wachsende und vielfältige Ansprüche gegenüber. Diese Feststellung ist nicht neu, die Probleme haben sich aber akzentuiert. Die Lösung dieser Knappheits- und Koordinationsprobleme und der damit verbundene Ausgleich von Nutzungsansprüchen zählen zu den raumplanerischen Grundanliegen.
Der Schweiz muß es gelingen, Wirtschafts- und Bevölkerungswachstum vom Flächenverbrauch zu entkoppeln. Das Verhalten, für neue Bedürfnisse, Ansprüche und Aufgaben stets wieder neues Land zu beanspruchen, ist nicht mehr vertretbar. Der Boden, die Fläche, der Raum wird noch viel zu wenig als nicht mehr vermehrbare und kaum erneuerbare Ressource betrachtet. Raumsparen muß in Zukunft ebenso populär werden wie beispielsweise das Energiesparen.

Raumsparen beinhaltet nicht nur quantitative Überlegungen bezüglich Ersparnis und Verbrauch, sondern auch qualitative Aspekte. Die schweizerische Kulturlandschaft hat sich nicht allein durch flächenmäßige Verschiebungen zwischen den verschiedenen Landnutzungen verändert, sie hat auch in ihrem Erscheinungsbild tiefgreifende Veränderungen erfahren. Es muß deshalb ebenfalls gelingen, daß Landschaftsbild und Landschaftscharakter möglichst wenig gefährdet und zerstört werden. Dabei geht es nicht allein um den Schutz besonders wertvoller Gebiete. Eine Politik, die einige wenige ausgewählte Gebiete möglichst gut schützt, die anderen aber mehr oder weniger ungeschützt läßt, ist abzulehnen. Beeinträchtigungen der Landschaft müssen überall möglichst vermieden oder gering gehalten werden.

Ferner muß es gelingen, mit dem Raumsparen auch im Siedlungsgebiet ernst zu machen. Siedlungserneuerung in städtischen (Stadterneuerung, Quartiererneuerung) und ländlichen (Dorferneuerung) Siedlungen müssen zügig an die Hand genommen werden. Dabei geht es nicht allein um Restaurierung, Renovierung, Umnutzung und Verdichtung, vielmehr geht es auch darum, die bestehenden Nutzungsmuster neu zu überdenken, um ein sinnvolles Nebeneinander der verschiedenen Nutzungen zu ermöglichen. Dies setzt voraus, daß die Verkehrs- und Siedlungsentwicklung besser aufeinander abgestimmt werden. Verkehrsmaßnahmen zählen zu den wichtigsten Maßnahmen der Siedlungsentwicklung und werden deshalb auf nationaler und lokaler Ebene nicht mehr als relativ isolierte Infrastrukturplanungen vollzogen. Die Frage, ob die Schweiz gebaut sei, ist folgendermaßen zu beantworten: «Die Schweiz ist heute wohl quantitativ gebaut, aber für die Bedürfnisse und Anforderungen von gestern. Ein Anpassen und Umrüsten des Gebäudeparks auf das qualitative Anforderungsprofil von heute und morgen verlangt große bauwirtschaftliche Leistungen auch weiterhin» (GABATHULER und WÜEST 1989, S. 5).

Bis anhin wurde versucht, Probleme der Enge mit Mitteln der Raumplanung zu lösen, Probleme der Weite mit solchen der Regionalpolitik (und der Raumplanung). Dies ist eine Folge der asymmetrischen schweizerischen Regionalpolitik, die sich auf die peripheren Regionen, die Berggebiete konzen- 
trierte. Die künftigen Probleme der räumlichen Ordnung und Organisation lassen sich jedoch nur lösen, wenn es gelingt, das sektorale Denken und Handeln innerhalb der Raumordnungspolitik zu überwinden; angesprochen sind nicht allein die Raumplanung und die Regionalpolitik, sondern alle raumrelevanten Politikbereiche. Zwischen wirtschaftlichen Interessen und anderen Ansprüchen an den Raum werden auch in Zukunft Spannungen bestehen. Diese werden sich sogar als Folge der zunehmenden Fremdbestimmung - die räumliche Ordnung und Organisation der Schweiz wird vermehrt von Entwicklungen geprägt, die außerhalb des schweizerischen Einflußbereiches liegen noch verstärken. Eine integrative, querschnittsorientierte Raumordnungspolitik wird in der Lage sein, solch räumliche Konflikte lösen zu helfen.

Wenn es der schweizerischen Raumordnungspolitik gelingt, aufzuzeigen, wie und vor allem auch daß räumliche Konflikte gelöst werden können, wird sie einen Beitrag leisten, der über die engen Grenzen der Schweiz von Bedeutung sein wird. Die Schweiz sollte den Ehrgeiz besitzen, eine für andere Länder und Regionen Europas beispielhafte Raumordnungspolitik $2000 \mathrm{zu}$ entwickeln. Dabei geht es nicht nur um «Know how», sondern auch um «Know what». Mit dem Beginn der Erarbeitung von Grundzügen der Raumentwicklung ist ein wichtiger erster Schritt getan worden.

\section{Schlußbemerkung}

Bei der Erarbeitung des Buches «Städte - wie wir sie wünschen» waren Schweizer Geographen maßgeblich mitbeteiligt. Es ist zu hoffen, daß bei der Er- arbeitung von Grundzügen der Raumentwicklung die Geographie ebenfalls ein entscheidendes Wort mitzusprechen hat und damit auch Mitverantwortung übernehmen kann. Die Geographie darf allerdings nicht warten, ob sie dazu eingeladen wird, vielmehr muß sie von sich aus die Initiative ergreifen, um in interdiszipliärer Zusammenarbeit die Zukunft des Lebensraumes Schweiz mitzugestalten.

\section{Literatur}

CAROL, H. und WERNER, M. (1949): Städte - wie wir sie wünschen, Zürich.

ELSASSER, H. (1990): Zehn Jahre Raumplanung in der Schweiz - Versuch einer Bilanz. In: Petermanns Geographische Mitteilungen, 134/2, 73-78.

GABATHULER, CHR. und WÜEST, H. (1989): Bauwerk Schweiz - Grundlagen und Perspektiven zum Baumarkt der 90er Jahre, Zürich.

KOLLER, A. (1990): Keine Revision des RPG! In: Bündner Vereinigung für Raumplanung Informationen, 4/90, 2-12.

Raumplanungskonferenz des Bundes (RPK) u. a. (Hrsg.) (1990): Welche Raumordnungspolitik brauchen wir in den goer Jahren? Bern.

RACINE, J.-B. et RAFFESTIN, C. (1990). Nouvelle Géographie de la Suisse et des Suisses, 2 vol., Lausanne.

SCHWEIZERISCHER BUNDESRAT (1987): Bericht über den Stand und die Entwicklung der Bodennutzung und Besiedlung in der Schweiz (Raumplanungsbericht 1987) vom 14. Dezember 1987, Bern.

SCHWEIZERISCHER BUNDESRAT (1987): Bericht über die Legislaturplanung 1987-1991 vom 18. Januar 1988, Bern.

SCHWEIZERISCHER BUNDESRAT (1989): Bericht über die Maßnahmen zur Raumordnungspolitik: Realisierungsprogramm vom 27. November 1989, Bern. 